\title{
Alteration in haemodynamics and pathological changes in the cardiovascular system during the development of Type 2 diabetes mellitus in OLETF rats
}

\author{
F. Saito, M. Kawaguchi, J. Izumida, T. Asakura, K. Maehara, Y. Maruyama \\ First Department of Internal Medicine, Fukushima Medical University, Fukushima city, Japan
}

\begin{abstract}
Aims/hypothesis. The process of cardiovascular complications in Type 2 diabetes mellitus (DM) is unclear. We investigated pathophysiological changes of the heart and vessels in the Otsuka Long-Evans Tokushima Fatty (OLETF) rat Type 2 DM model during a long time period.

Methods. Echocardiography was carried out at 22 and 62 weeks of age of OLETF ( $n=10$, each) and agematched Long-Evans Tokushima Otsuka (LETO) rats $(n=10$, each) as a reference. Haemodynamic measurements and histological examinations of the heart and the coronary and aortic vascular walls were done.

Results. The left ventricular (LV) maximal -dP/dt was reduced in OLETF rats at 62 weeks $(-1085 \pm 35 \mathrm{mmHg} / \mathrm{s})$ less than that at 22 weeks $(-1892 \pm 396 \mathrm{mmHg} / \mathrm{sec}$, $p<0.05)$ and in LETO rats at 62 weeks $(-1306 \pm 200$
\end{abstract}

$\mathrm{mmHg} / \mathrm{sec}, p<0.05)$. Wall thickening of intramyocardial coronary arteries, capillary tortuosity and thickening of basement membrane were evident in OLETF rats at 62 weeks. Intimal and medial wall thickening of the aorta were prominent in OLETF rats at 62 weeks $(15 \pm 2.2$ and $90 \pm 6.6 \mu \mathrm{m}$, in LETO rats at 62 weeks, $2 \pm 0.4$ and $65 \pm 5.2 \mu \mathrm{m}, p<0.05$, and in OLETF rats at 22 weeks, $7 \pm 4.6$ and $71 \pm 6.0 \mu \mathrm{m}, p<0.05$, respectively).

Conclusions/interpretation. In the Type 2 DM model, angiopathy, especially in coronary arteries including small vessels, as well as a LV relaxation abnormality, are induced in a late stage of DM. These are considered to be important complications in Type $2 \mathrm{DM}$. [Diabetologia (2003) 46:1161-1169]

Keywords Diabetes mellitus, Otsuka-Long-Evans Tokushima Fatty rat, coronary heart disease, cardiac function, atherosclerosis.
Received: 3 December 2002 / Revised: 6 February 2003

Published online: 11 July 2003

C) Springer-Verlag 2003

Corresponding author: Dr. Y. Maruyama, First Department of Internal Medicine, Fukushima Medical University, Hikarigaoka-1, 960-1295 Fukushima city, Japan

E-mail: maruyama@fmu.ac.jp

Abbreviations: OLETF, Otsuka Long-Evans Tokushima Fatty; DM, diabetes mellitus; LETO, Long-Evans Tokushima Otsuka; STZ, streptozotocin; 2-DE, two-dimensional echocardiography; LVDd, left ventricular end-diastolic diameter; LVDs, left ventricular end-systolic diameter; IVST, diastolic intraventricular septal wall thickness; LVPWT, left ventricular posterior wall thickness; LVEF, left ventricular ejection fraction; LV, left ventricle; $\mathrm{dP} / \mathrm{dt}$, first derivative of LV pressure.
In Type 1 and 2 diabetes mellitus (DM) left ventricular hypertrophy and failure, known as diabetic cardiomyopathy $[1,2,3]$, could develop and morbidity from chronic cardiac failure in DM is high [4]. The risk of coronary heart disease (CHD) is also high and is about the same in Type 1 and Type 2 DM [5], suggesting epicardial coronary artery disease. However, the pathological changes in the vascular system of human diabetic (Type 1 and 2) hearts have been shown mainly in capillary vessels $[6,7,8]$.

Information from DM patients is important for understanding DM-induced pathological changes but clinical findings are frequently modified by various DM-dependent and -independent complications. In contrast, information from animal DM models is useful, although extrapolation to clinical situations must be done with care. In experimental studies using al- 
loxan or streptozotocin (STZ)-induced diabetes, i.e., a Type 1 DM model, systolic and diastolic left ventricular function and pathological changes of the myocardium have been reported $[9,10,11,12,13,14,15]$. However, there is little evidence of atherosclerosis or pathological changes of small coronary arteries in such Type 1 diabetic animals [16].

In contrast, there is little experimental data about the cardiovascular system in Type $2 \mathrm{DM}$, because a suitable model of Type $2 \mathrm{DM}$ has not been available until recently when the Otsuka-Long-Evans Tokushima Fatty (OLETF) rats were developed as an animal model of Type 2 DM [17, 18, 19, 20, 21]. OLETF rats are characterized by: (i) increasing body weight just after weaning, in contrast to STZ-induced DM rats, (ii) late onset of hyperglycaemia (after 18 weeks of age) and diagnosable DM by OGTT after 24 weeks of age, (iii) hyperinsulinaemia at 24 weeks of age and its decline after 55 weeks of age, and (iv) increasing plasma cholesterol and triglyceride concentrations after 21 weeks of age $[17,18]$.

An important question about Type $2 \mathrm{DM}$ is whether the sequence of cardiovascular complications leading to cardiac dysfunction during the development of diabetes differs from that of Type 1 DM. Thus, we examined the pathological changes, if any, in coronary blood vessels and myocardial dysfunction in OLETF rats. We investigated cardiac function and histopathological alterations of the coronary artery and aorta at the early (22 weeks, matured) and late (62 weeks, aged) phases of Type 2 DM in OLETF rats [21], and compared them to those of age-matched Long-Evans Tokushima (LETO) rats, which do not develop DM $[17,20]$.

\section{Materials and methods}

All protocols were in accordance with the Guideline on Animal Experiments of Fukushima Medical University and the Japanese Government Animal Protection and Management Law (No. 115).

Experimental animals. We used five-week-old male OLETF rats $(n=20)$ and LETO rats $(n=20)$ as controls (Otsuka Pharmaceutical, Tokushima, Japan), since they do not develop DM or impaired glucose tolerance by OGTT [17]. All rats were caged individually and received normal rat chow and tap water ad libitum in a controlled environment [room temperature $23 \pm 1.5^{\circ} \mathrm{C}$, room humidity $55 \pm 5 \%$ ), until 19 to 24 weeks (mean, 22 weeks) old, or 59 to 64 weeks (mean, 62 weeks) old (10 OLETF and 10 LETO rats) respectively].

Experimental protocol. All rats were anaesthetized by intraperitoneal administration of sodium pentobarbital $(50 \mathrm{mg} / \mathrm{kg})$. Blood was sampled from the tail vein for measurement of casual blood glucose concentration using a Glucose Analyzer (Daikin, Antsense II, Osaka, Japan). Thereafter, two-dimensional echocardiography (2-DE) was carrried out in a prone position.

After the 2-DE measurement, the chest was opened under artificial ventilation, and haemodynamic variables were mea- sured as described below. After the experiment, the heart was isolated after perfusion fixation at a pressure of $100 \mathrm{mmHg}$. Histopathological and immunohistochemical examinations were done after the relevant treatment.

Transthoracic 2-DE. Two-dimensional echocardiography (2-DE) was carried out using an echocardiographic system equipped with a $10-\mathrm{MHz}$ transducer (Hewlett-Packard, SONOS 100CF, Andover, Mass., USA). Heart rate, left ventricular end-diastolic (LVDd) and end-systolic diameter (LVDs), diastolic intraventricular septal wall thickness (IVST), and diastolic left ventricular posterior wall thickness (LVPWT) were measured. Values of five consecutive beats were averaged. Left ventricular ejection fraction (LVEF) was calculated using the Pombo method [22], with the following formula:

$\operatorname{LVEF}(\%)=\left[\left(\operatorname{LVDd}^{3}-\operatorname{LVDs}^{3}\right) / \operatorname{LVDd}^{3}\right] \times 100$.

In vivo haemodynamic measurements. After the 2-DE measurement, needle electrodes were inserted into each limb for recording an electrocardiogram (ECG). A thoracheotomy was done and a tracheal tube (18-gauge venula) was inserted. Artificial ventilation was then started, using a rodent respirator (SHIMANO, respirator MODEL-SN-480-7,Tokyo, Japan) and the chest was opened. A 23-gauge needle was inserted from the apex into the left ventricle (LV) and connected to a pressure transducer (Nihon Kohden, TP4005, Tokyo, Japan) for the measurement of LV peak pressure, LV end-diastolic pressure, and the first derivative of $\mathrm{LV}$ pressure $(\mathrm{dP} / \mathrm{dt})$. ECG (lead II) as well as other variables which were all continuously monitored and recorded on a thermal array recorder polygraph system (Nihon Kohden, Polygraph System, Tokyo, Japan) at a paper speed of $100 \mathrm{~mm} / \mathrm{sec}$. LV pressure and $\mathrm{dP} / \mathrm{dt}$ were averaged for ten consecutive beats. Rate pressure product (RPP) was calculated from heart rates and LV systolic pressure.

Histopathological examination. Perfusion fixation with $4 \%$ paraformaldehyde solution was done in five rats from each group at a constant perfusion pressure of $100 \mathrm{mmHg}$ using the needle inserted into the LV cavity. Then, the heart and thoracic aorta were isolated and removed, and the heart was weighed. Both ventricles were cut transversely parallel to the atrio-ventricular groove at the equatorial plane. The hearts and aortas were embedded in paraffin and sectioned. All sections $(4 \mu \mathrm{m}$ thick) were stained with haematoxylin-eosin and Azan stains (Mallory-Heidenhain) for connective tissues. Pictures of heart tissues were taken at 200-fold magnification, and the parameters described below were measured with a photomicroscope analysis system. For evaluating wall thickening of the arteries, the wall-to-lumen ratios from arterioles to large epicardial arteries, diameters which were from $10 \mu \mathrm{m}$ to $300 \mu \mathrm{m}$, were calculated using the following formula: $(\mathrm{O}-\mathrm{I}) / \mathrm{I}$, where $\mathrm{O}$ and $\mathrm{I}$ are the diameters of the areas encircled by the outer and inner borders of the vessels [23]. In each heart, 20 to 30 microvessels and three to ten epicardial vessels were examined. For the thoracic aorta, the widths of the intimal and medial layers were measured separately.

In each section, transverse diameters of myocytes, in which the nucleus appeared, were measured from the epicardium to the endocardium of the LV free wall at the mid ventricular level. The values from 50 myocytes were averaged.

Immunohistochemical staining for type III collagen, type IV collagen, and laminin. We carried out semi-quantitative analyses of type III collagen contribution to interstitial fibrosis, and type IV collagen and laminin contributions to the base- 
Table 1. Body weight, plasma glucose concentration, and heart rate in OLETF and LETO rats at 22 and 62 weeks

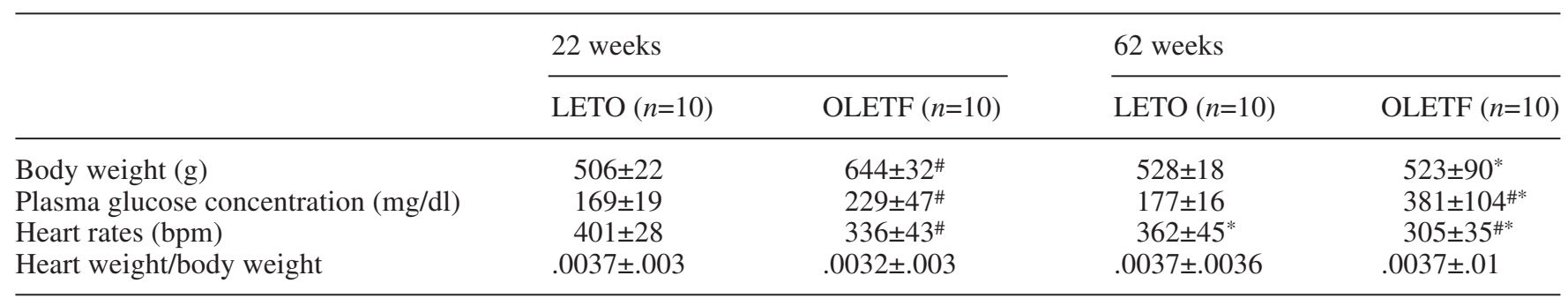

Data are means \pm SD

$* p<0.05$ vs. 22 weeks in the same experimental group

$\# p<0.05$ vs. LETO rats in the same experimental periods

Table 2. Two-dimensional echocardiography findings and haemodynamic variables

\begin{tabular}{|c|c|c|c|c|}
\hline & LETO $(n=10)$ & $\operatorname{OLETF}(n=10)$ & LETO $(n=10)$ & OLETF $(n=10)$ \\
\hline $\operatorname{LVDd}(\mathrm{mm})$ & $6.6 \pm 0.7$ & $6.4 \pm 0.3$ & $6.8 \pm 0.4$ & $6.8 \pm 0.8$ \\
\hline LVDs (mm) & $3.3 \pm 0.6$ & $3.1 \pm 0.4$ & $3.2 \pm 0.2$ & $3.1 \pm 0.8$ \\
\hline IVST (mm) & $1.5 \pm 0.4$ & $1.4 \pm 0.2$ & $1.7 \pm 0.2$ & $1.7 \pm 0.3$ \\
\hline LVEF (\%) & $85 \pm 4$ & $88 \pm 3$ & $88 \pm 5$ & $86 \pm 3$ \\
\hline \multicolumn{5}{|l|}{ Haemodynamics } \\
\hline LVSP (mm Hg) & $98 \pm 17$ & $95 \pm 18$ & $74 \pm 5 *$ & $73 \pm 8^{*}$ \\
\hline $\mathrm{RPP}(\mathrm{mmHg} / \mathrm{min})$ & $39479 \pm 8065$ & $32318 \pm 7888$ & $27463 \pm 6630 *$ & $23252 \pm 4129 *$ \\
\hline LVEDP (mm Hg) & $8 \pm 4$ & $8 \pm 1$ & $8 \pm 2$ & $8 \pm 1$ \\
\hline Maximal $+\mathrm{dP} / \mathrm{dt}$ & $2681 \pm 418$ & $2680 \pm 311$ & $2141 \pm 475^{*}$ & $2238 \pm 113 *$ \\
\hline
\end{tabular}

Data are means \pm SD. ${ }^{\#} p<0.05$ vs. LETO rats in the same experimental period. ${ }^{*} p<0.05$ vs. 22 weeks in the same experimental group. LVDd, left ventricular end diastolic dimension; LVDs, left ventricular end systolic dimension; IVST, diastolic

ment membrane of coronary vascular beds, using immnohistochemical staining techniques. From each heart five paraffin sections were deparaffinized and after processing with alcohol, immunoperoxidase staining was done by the avidin-biotin-peroxidase complex method [24]. Sections were quenched at room temperature for $30 \mathrm{~min}$ in methanol containing $3 \%$ $\mathrm{H}_{2} \mathrm{O}_{2}$, washed for $5 \mathrm{~min}$ in tap water and then incubated for $1 \mathrm{~h}$ in pepsin solution. The sections were incubated at $4^{\circ} \mathrm{C}$ overnight with specific type III collagen, type IV collagen and laminin antiserum (Fuji Chemical Industrial, Tokyo, Japan) diluted 300 -fold with an antibody diluting buffer. After washing with a phosphate buffer solution, the sections were incubated at room temperature for 30 min with an anti rabbit IgG biotinylated antibody (Nichirei, Tokyo, Japan). Reactions were visualized by incubation in 3,3'-diaminobenzidine solution for $5 \mathrm{~min}$ and counterstaining with Masson modified haematoxylin for $3 \mathrm{~min}$. Negative controls consisted of replacing the primary antibody with non-immune rabbit serum.

Electron microscopy. In each age group five OLETF and LETO rats were fixed by perfusion with $2 \%$ glutaraldehyde as described above. The hearts and thoracic aortas were then isolated and removed, after this a part of the tissues was postfixed with $1 \%$ osmium tetroxide. The tissues were passed through a graded alcohol series followed by propylene oxide, and embedded in Epon 812. Ultrathin sections were stained with ura- intraventricular septal wall thickness; LVPWT, left ventricular posterior wall thickness; LVEF, left ventricular ejection fraction; LVSP, left ventricular systolic pressure; RPP, rate pressure product; LVEDP, left ventricular end diastolic pressure

nyl acetate and lead citrate, and examined by transmission electron microscopy (JEOL-1200 EX, Tokyo, Japan).

Statistical analysis. Data are expressed as means \pm SD. The significance of differences in haemodynamic variables and 2-DE parameters between LETO and OLETF rats at each experimental period and between different periods was determined using one-way ANOVA followed by the Student's $t$ test. Differences in vessel thickening corresponding to the difference in vessel lumen diameters were evaluated by one-way ANOVA followed by the Fischer exact test for multiple comparisons. The Spearman correlation was used to investigate the relation between vessel diameter and vessel thickening in coronary arteries. A $p$ value of less than 0.05 was considered statistically significant.

\section{Results}

General features. At 22 weeks the body weight of OLETF rats was greater than that of LETO rats $(644 \pm 32 \mathrm{~g}$ vs $506 \pm 22 \mathrm{~g}, p<0.05)$ (Table 1). However, the body weight of OLETF rats at 62 weeks was less than that at 22 weeks (62 weeks, $523 \pm 90 \mathrm{~g}, p<0.05$ ). Casual blood glucose concentrations in the OLETF 


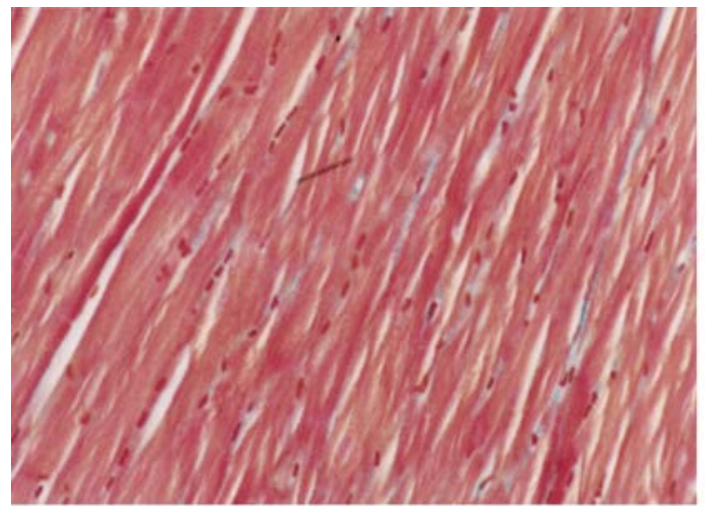

LETO rat

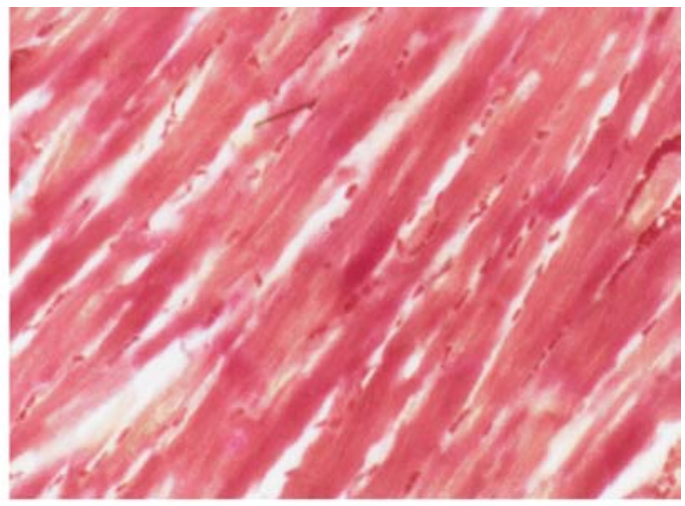

OLETF rat

\section{Azan staining $\mathrm{x} 200$}

Fig. 1. Azan staining of cardiac myocytes at 62 weeks $(\times 200)$. The transverse diameter of cardiac myocytes was not different in LETO and OLETF rats
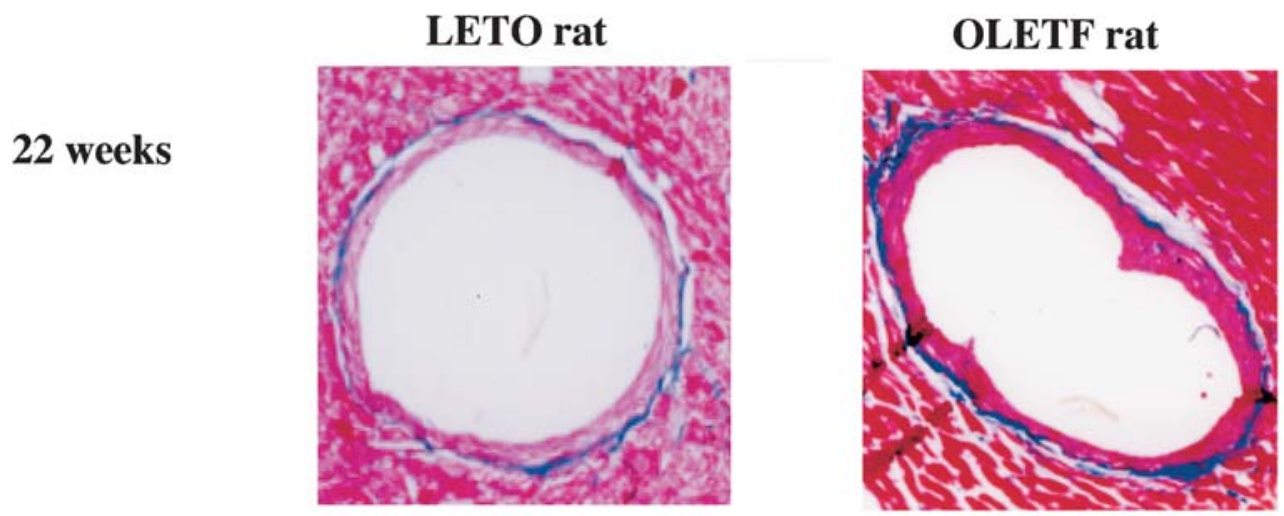

\section{2 weeks}
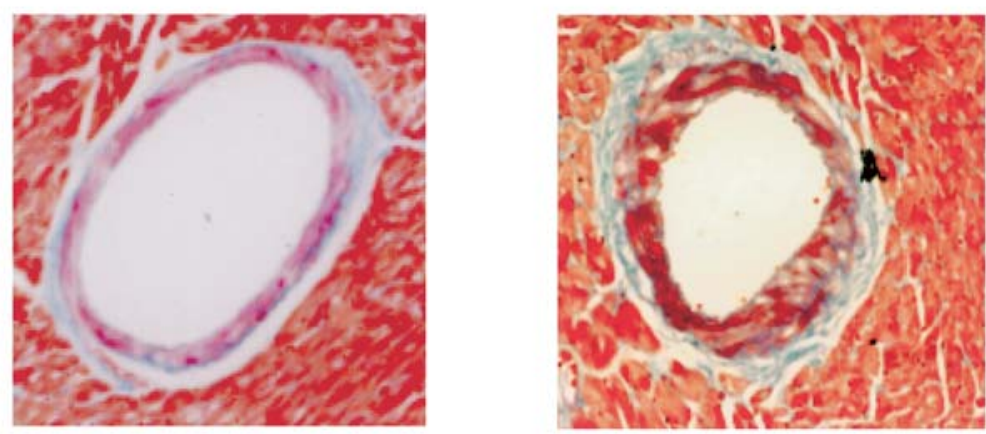

\section{$100 \mu \mathrm{m}$}

Azan staining

Fig. 2. Azan staining of coronary artery in the cardiac tissue of OLETF and LETO rats at 22 weeks (upper panel) and 62 weeks (lower panel) of age. The interstitial collagen fibres tended to increase in connective tissues around vessels in both OLETF and LETO rats at 64 weeks, but there was no significant difference between the two groups. Thickening of the wall was observed in OLETF rats at 62 weeks $(\times 200)$

rats were higher than in the LETO rats at each period ( 22 weeks: $229 \pm 47 \mathrm{mg} / \mathrm{dl}$ vs $169 \pm 19 \mathrm{mg} / \mathrm{dl}, 62$ weeks: $381 \pm 104 \mathrm{mg} / \mathrm{dl}$ vs $177 \pm 16 \mathrm{mg} / \mathrm{dl}, p<0.05$, respective1y), and casual blood glucose concentrations of
OLETF rats at 62 weeks were higher than at 22 weeks $(p<0.01)$. Heart rates were different at 22 weeks and 62 weeks in both LETO and OLETF rats, and heart rates of OLETF rats were lower than those of LETO rats at each period $(p<0.05)$.

Haemodynamic variables. LVEF, LVDd, and LVDs were not different in OLETF and LETO rats at each period (Table 2). LV end-diastolic pressure and LV peak systolic pressure as well as LV maximal $+\mathrm{dP} / \mathrm{dt}$ were also not different in LETO and OLETF rats at 22 and 62 weeks. However, LV maximal $-\mathrm{dP} / \mathrm{dt}$ was 
smaller in OLETF rats $(-1085 \pm 35 \mathrm{mmHg} / \mathrm{s})$ than in LETO rats $(-1306 \pm 200 \mathrm{mmHg} / \mathrm{s})$ at 62 weeks $(p<0.05)$. Moreover, LV peak systolic pressure and LV maximal $+\mathrm{dP} / \mathrm{dt}$ in LETO and OLETF rats were lower at 62 weeks $(74 \pm 5 ; 73 \pm 8 \mathrm{mmHg}$ and $2,141 \pm 475$; $2238 \pm 113 \mathrm{mmHg} / \mathrm{s})$ than at 22 weeks $(98 \pm 17$; $95 \pm 18 \mathrm{mmHg}$, and $2681 \pm 418 ; 2680 \pm 311 \mathrm{mmHg} / \mathrm{s}$, $p<0.05$, respectively). RPP were reduced in LETO and OLETF rats at 62 weeks $(27,463 \pm 6630 \mathrm{mmHg} / \mathrm{min}$ and $23,252 \pm 4129 \mathrm{mmHg} / \mathrm{min}$ ) than at 22 weeks $(37479 \pm 8065 \mathrm{mmHg} / \mathrm{min}$ and $32,318 \pm 7888 \mathrm{mmHg} /$ min, $p<0.05$, respectively).

Table 3. The diameter of myocytes $(\mu \mathrm{m})$

\begin{tabular}{lll}
\hline & LETO & OLETF \\
\hline 22 weeks & $12.2 \pm 1.2$ & $11.6 \pm 1.4$ \\
62 weeks & $11.4 \pm 1.8$ & $11.4 \pm 1.4$ \\
\hline
\end{tabular}

There was no significant differences

There were five rats in each group, and 50 cells were counted in each rat
Morphological findings. The transverse diameters of cardiac myocytes were not different in LETO and OLETF rats at 22 and 62 weeks (Fig. 1 and Table 3). The interstitial collagen fibres tended to increase in connective tissues around the small coronary arterial vessels in both LETO and OLETF rats at 62 weeks compared to 22 weeks. However, there was no difference in the amount of interstitial collagen fibres between LETO and OLETF rats at both 22 and 62 weeks. Thickening of vessel wall was observed in OLETF rats at 62 weeks (Fig. 2). The wall-to-lumen ratio with respect to vessel diameters in OLETF and LETO rats at each experimental period are shown in scatterplots. The wall-to-lumen ratio increased in small coronary arteries and arterioles only in OLETF rats at 62 weeks. There was a correlation between the wall-to-lumen ratios and the diameters $(p<0.001)$ in OLETF rats at 62 weeks (Fig. 3 ). The average wall-tolumen ratios in coronary arterioles with diameters less than $100 \mu \mathrm{m}$ were greater in OLETF rats $(3.70 \pm 2.8$, measured samples $n=82)$ than LETO rats $(0.89 \pm 0.61$, measured samples $n=55, p<0.0001)$ at 64 weeks. The average wall-to-lumen ratios in coronary arteries and
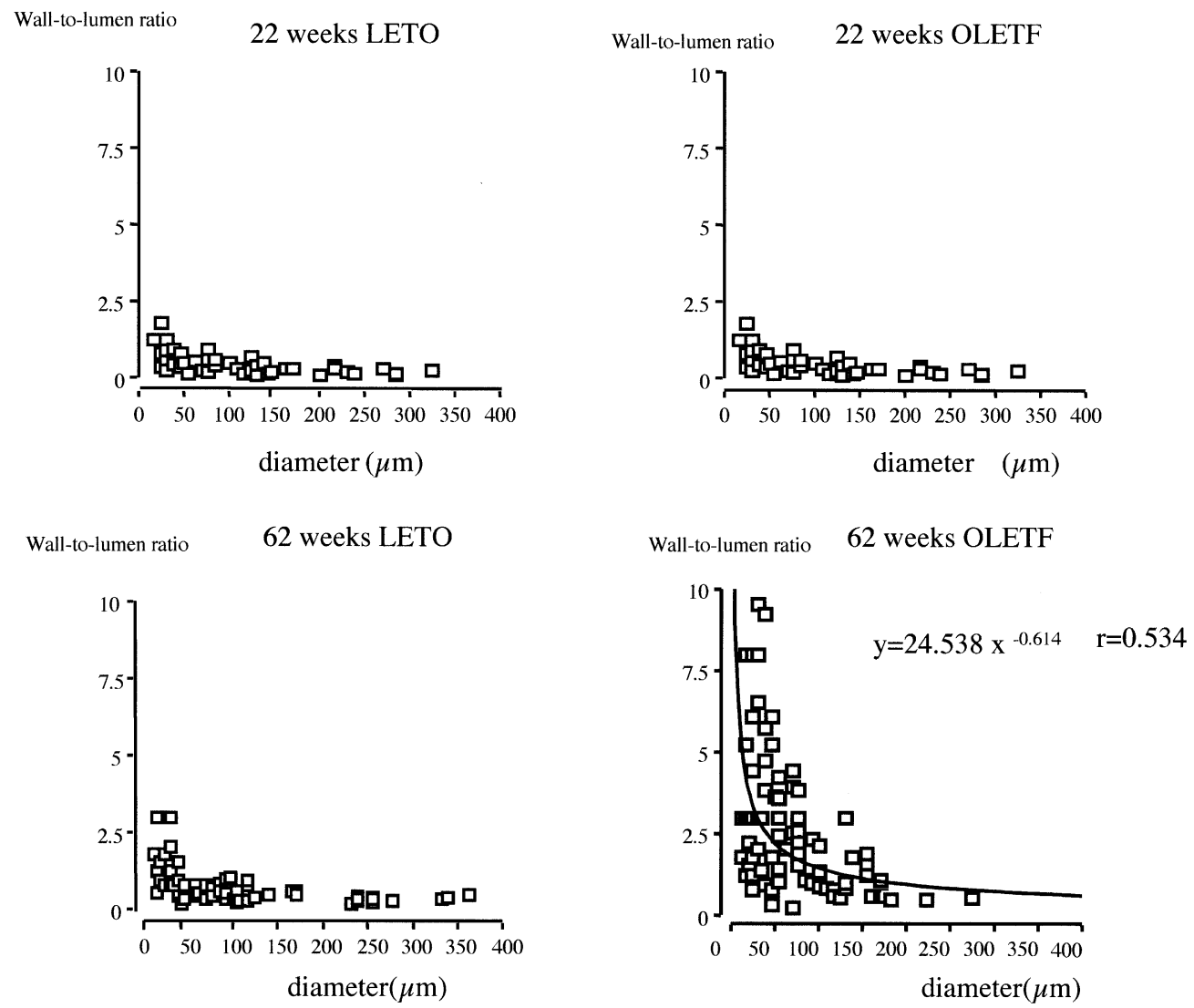

arteries of diameters from 10 to $350 \mu \mathrm{m}$. However, in OLETF rats, the relationships became greater as the vessel diameters became smaller, and there was a correlation between the wallto-lumen ratios and the vessel diameters $\left(y=24.538 \mathrm{x}^{-0.614}\right.$, $\mathrm{r}=0.534, p<0.0001$ ) 
arterioles with diameters of $100 \mu \mathrm{m}-350 \mu \mathrm{m}$ were also larger in OLETF rats (LETO $0.44 \pm 0.18, n=20$; OLETF $1.04 \pm 0.68, n=17, p=0.006)$.

The thickness of intima and media also increased in thoracic aorta only in OLETF rats at 62 weeks (Table 4). At 22 weeks, the thickness of aortic wall was not different in LETO and OLETF rats. However, the intimal and medial wall thickenings in OLETF rats $(15 \pm 2.2 \mu \mathrm{m}$ and $90 \pm 6.6 \mu \mathrm{m}$, respectively) were greater than in LETO rats $(2 \pm 0.4 \mu \mathrm{m}$ and $65 \pm 5.2 \mu \mathrm{m}, p<0.05$ vs OLETF rats, respectively) at 62 weeks. Infiltrations of monocytes and macrophages were observed in the intima of the thoracic aorta in 62 weeks OLETF rats at 62 weeks (Fig. 4).

Immunohistological findings. There were no differences in the degrees of staining of type III collagen, type IV collagen, and laminin in OLETF and LETO

Table 4. The intimal and medial wall thickness of thoracic aorta in OLETF and LETO rats

\begin{tabular}{llrc}
\hline & & 22 weeks & 62 weeks \\
\hline \multirow{2}{*}{ LETO } & Intima $(\mu \mathrm{m})$ & $2 \pm 0.4$ & $2 \pm 0.4$ \\
& Media $(\mu \mathrm{m})$ & $62 \pm 4.0$ & $65 \pm 5.2$ \\
\multirow{2}{*}{ OLETF } & Intima $(\mu \mathrm{m})$ & $7 \pm 4.6$ & $15 \pm 2.2^{\# *}$ \\
& Media $(\mu \mathrm{m})$ & $71 \pm 6.0$ & $90 \pm 6.6^{\# *}$ \\
\hline
\end{tabular}

${ }^{\#} p<0.05$ vs. LETO rats in the same experimental period ${ }^{*} p<0.05$ vs. 22 weeks in the same experimental group $n=5$ in each group, and 30 points were calculated in each rat

\section{A. LETO rat at 62 weeks}

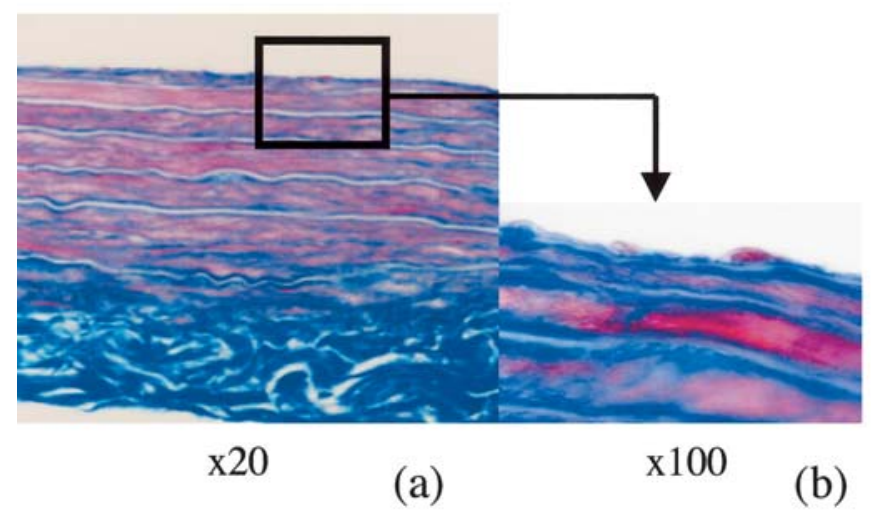

Fig. 4A, B. Azan staining of the thoracic aorta at 62 weeks of age of LETO (A) and OLETF $(\mathbf{B})$ rats. Intima and medial wall hypertrophy, accompanied with infiltration of monocytes under the endothelial cells (Arrow) and atheromatous plaque were observed (B-b). It was observed that a monocyte (large arrow) infiltrated under an endotherial cell (small arrow) by electron microscopy (B-c) rats at 24 and 62 weeks. However, a coil-like meandering of capillary vessels was observed in OLETF rats at 62 weeks (Fig. 5, upper panel).

Electron microscopic examination. Capillary basement membrane thickening in the heart appeared in OLETF rats at 62 weeks, as shown in figure 5 (lower panel) and Table $5(177 \pm 66 \mathrm{~nm}$ vs $87 \pm 12 \mathrm{~nm}$, $p<0.05)$.

\section{Discussion}

This study investigated LV function, assessed by echocardiography and haemodynamics, and histopathological changes of myocardium and the vascular system, in a Type 2 diabetic animal model with long survival. The main results obtained with OLETF rats are as follows: (i) Functional and pathological changes of the LV myocardium were not observed, except

Table 5. Capillary basement membrane thickening of the heart measured by electron microscopy $(\mathrm{nm})$

\begin{tabular}{lll}
\hline & LETO $(n)$ & OLETF $(n)$ \\
\hline 22 weeks & $90 \pm 12(50)$ & $106 \pm 20(50)$ \\
62 weeks & $87 \pm 12(50)$ & $177 \pm 66(50)^{\# *}$ \\
\hline
\end{tabular}

All data are means \pm SD. Abbreviations are the same as in Table 4

Five rats were prepared in each group

\section{B. OLETF rat at 62 weeks}
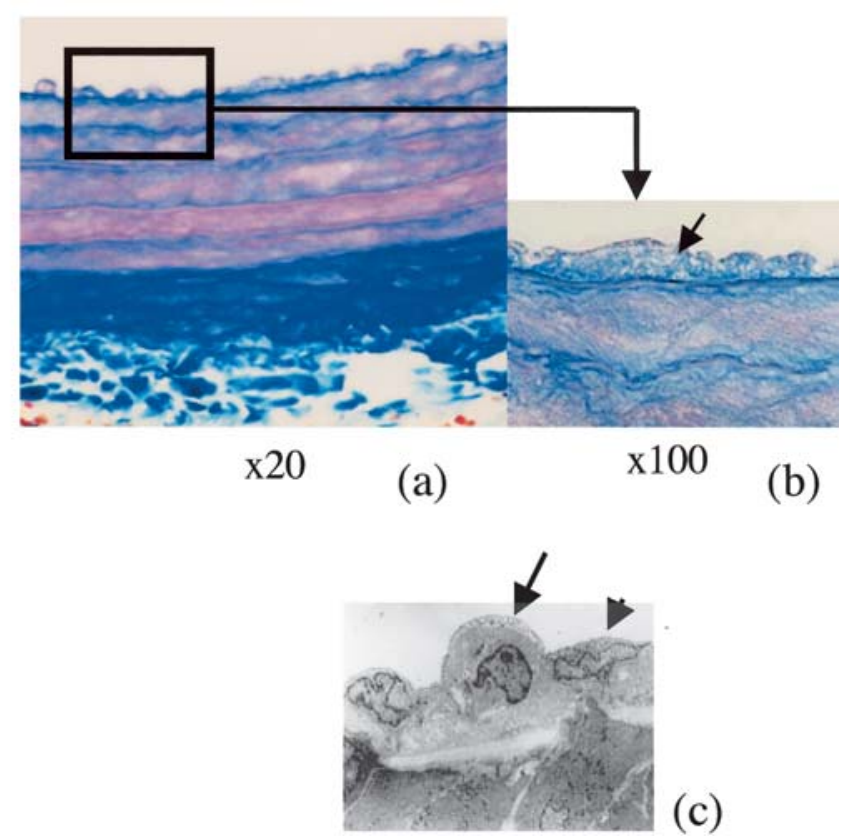
LETO rat at 62 weeks

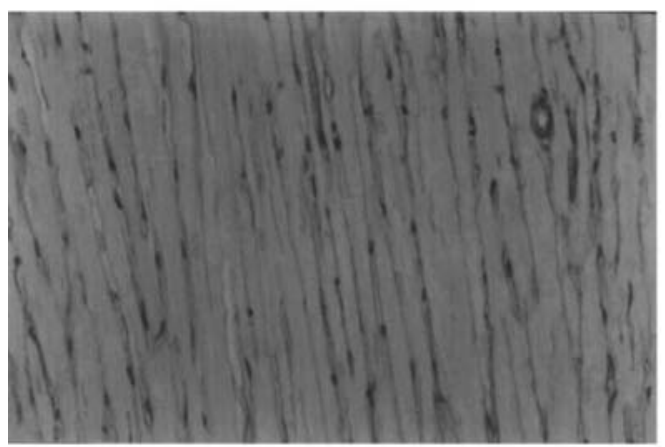

Capillary vessels
OLETF rat at 62 weeks

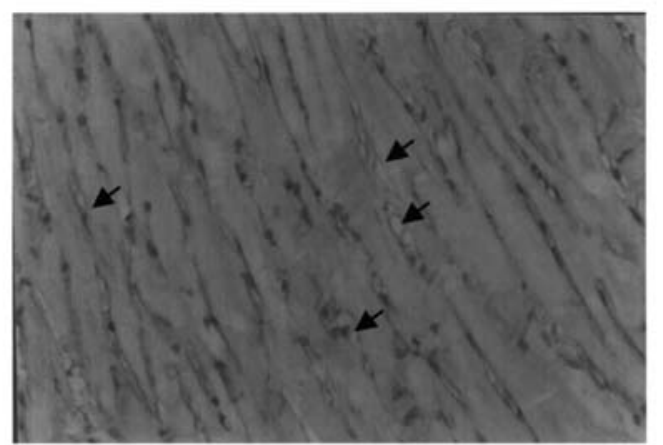

Capillary vessels

$<$ Type IV collagen staining >

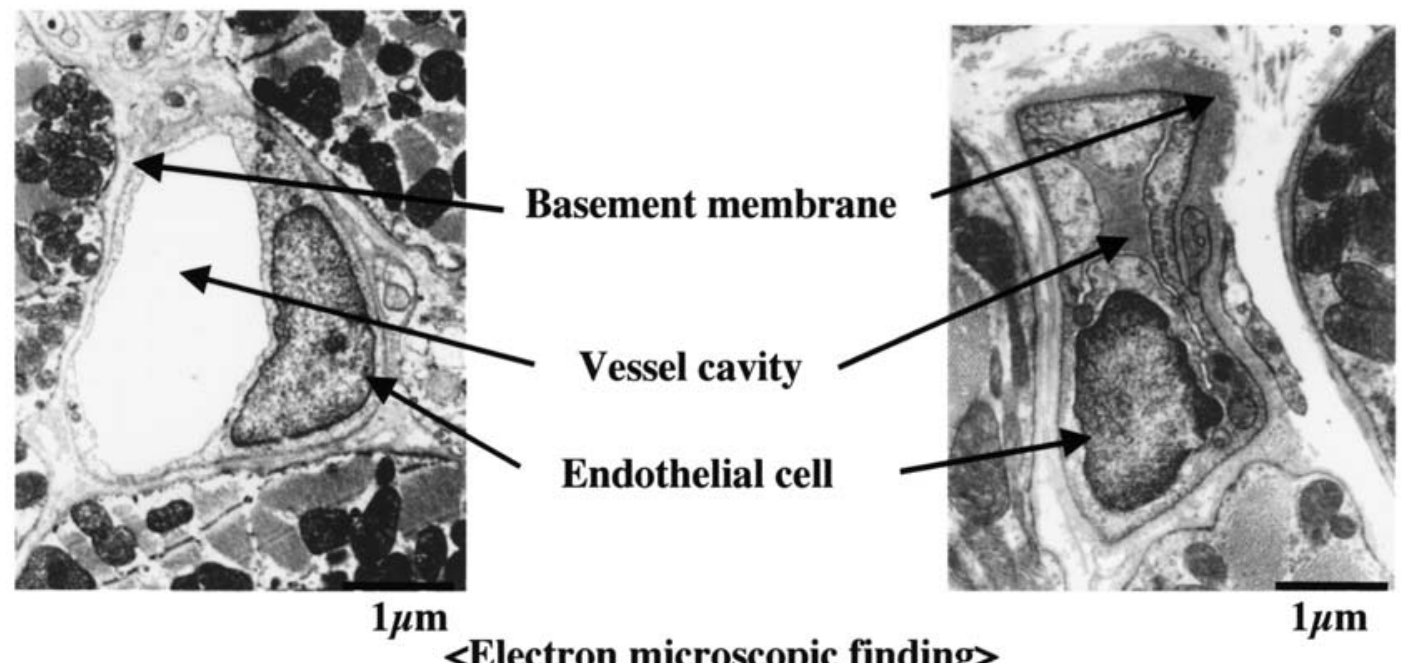

Fig. 5. Upper panel: LV staining for immunohistochemistry of collagen type IV in OLETF and LETO rats at 62 weeks of age. The capillary vessels in the heart changed spirally (arrow) in OLETF rats $(\times 200)$. Lower panel: Electron micrograph showing capillary basement membrane in the heart was thickened in OLETF rats at 62 weeks

for a relaxation abnormality in older OLETF rats at 62 weeks. The size of cardiomyocytes, and the amount of interstitial collagen were not different, compared to LETO rats. (ii) The intimal and medial walls of the thoracic aorta were thickened with infiltration of monocytes under endothelial cells or in the plaque formation only in older OLETF rats at 62 weeks. (iii) Wall thickening of intramyocardial small coronary arteries appeared only in older OLETF rats at 62 weeks and the degree of wall thickening was prominent as the vessel diameters became small. Capillary tortuosity and thickened capillary basement membrane was found in older OLETF rats at 62 weeks. These findings were considered to be important complications in long lasting Type 2 DM.

Myocardial dysfunction, such as a decrease in the contractile performance of the papillary muscle fibre or of the isolated perfused heart, has been well docu- mented in STZ or alloxan-induced DM rats (Type 1 DM model) $[9,10,11,12,13,14,25,26]$. However, in this study LV ejection fraction, LV peak systolic pressure and maximal $+\mathrm{dP} / \mathrm{dt}$ did not change in OLETF rats compared to LETO rats, although LV peak systolic pressure and LV maximal $+\mathrm{dP} / \mathrm{dt}$ in both LETO and OLETF at 62 weeks in the open chest state decreased similarly compared to the rats at 22 weeks. Among the indices of LV function only maximal - $\mathrm{dP} / \mathrm{dt}$ decreased in OLETF rats compared to LETO rats, suggesting impaired myocardial relaxation [27]. Relaxation abnormality in the diabetic heart has been found in clinical studies as well as experimental investigations [28, 29]. Another study [30] recently reported that an increase of interstitial collagen and diastolic filling abnormalities appeared in the pre-diabetic stage in OLETF rats. However, in the diabetic stage at 47 weeks of age, the amount of interstitial collagen and LV diastolic function in OLETF rats was not different from LETO rats.In contrast, no collagen accumulation in the extracellular matrix of the heart or LVEDP and LVDd abnormalities were found in this study. The reason for this difference between the two studies is unclear. Although the cause of relaxation abnormality is not clarified by this study, it is suggested that depressed myofibrillar $\mathrm{Ca}^{2+}$-stimulated ATPase, de- 
creased sarcoplasmic reticulum $\mathrm{Ca}^{2+}$-pump activities, and abnormal sympathetic innervation of the heart might be partly responsible for impaired relaxation of the diabetic heart $[8,31]$. Moreover, changes in the afterload resistance might partially relate to lower maximal - $\mathrm{dP} / \mathrm{dt}$ at 62 weeks in OLETF rats, although afterload resistance was not measured in this study. LV$\mathrm{dP} / \mathrm{dt}$ might also be altered by changes in various factors such as temperature, plasma or tissue catecholamines, and heart rate. Thus, this issue remains to be clarified. In our study, heart rate in OLETF rats decreased compared to LETO rats. It is of interest that heart rate decreased in DM rats, since diabetic cardiomyopathy is expected to also be associated with heart neuropathy, showing decreased parasympathetic modulation and higher heart rates. In contrast to this possibility, decreased heart rate has been reported in STZinduced DM rats [9]. The underlying mechanism of the association of diabetes and reduced heart rates is unclear although it could be partly due to changes in the electrophysiological properties of the sino-atrial node as indicated in isolated hearts by a depressed spontaneous activity of the pacemaker [32] and/or to decreased sensitivity to adrenergic stimulation, or increased sensitivity to muscarinic stimulation [9]. However, these previous results were obtained using STZ-induced Type 1 DM rats, so further study of the Type 2 DM condition is needed to explain the alteration of heart rates in diabetic cardiomyopathy associated with heart neuropathy.

One study [33] reported that in OLETF rats at 30 weeks there was perivascular fibrosis in the LV, and LV mRNA levels for collagen types I, III and IV, laminin, and TGF- $\beta$ in extracellular matrix were higher than in LETO rats. However, at 54 weeks the degree of LV interstitial fibrosis and LV mRNA levels for collagen type I, III, and IV, and laminin were not different from the age-matched LETO rat. The lack of significant interstitial fibrosis and perivascular fibrosis in OLETF rats at 62 weeks in this study is consistent with their results [33] obtained at 54 weeks, although we did not examine type I collagen or TGF- 3 . However, it should be noted that in this study OLETF rats at 22 weeks also did not show interstitial fibrosis or changes in type III and IV collagen. Further study is needed to determine whether this is due to the very early stage of Type $2 \mathrm{DM}$, or whether those changes are observed only in a specific stage of Type $2 \mathrm{DM}$.

The most prominent pathological findings in this study with OLETF rats were aortic atherosclerosis and wall thickening of small coronary arteries. Monocyte infiltration and development of the atherosclerotic process could be partly associated with these vascular changes. As far as we know these findings in a Type 2 DM model are the first to show the pathological changes in the important arterial system. Capillary tortuosity and thickening of capillary basement membrane in the heart, as described previously in Type 1 and 2
DM [34], were also observed in our OLETF rat model. In contrast, in this study the pathological changes in the epicardial coronary artery seemed to be small during the observation period. Despite the fact that atherosclerotic changes and/or vascular injury in aorta and small coronary arteries, including capillary vessels, are prominent in the OLETF rat Type 2 DM model, serious myocardial damage or ventricular re-modelling was not clearly observed. However, we cannot rule out the possibility that vascular changes in the aorta and coronary arterial system could lead to severe cardiac re-modelling and dysfunction after a longer period of observation, because cardiovascular complications are without doubt the most common cause of death in diabetic patients, with no apparent difference between Type 1 and 2 DM [35]. As far as we know, there are no previous data concerning the cardiac pathophysiologial and vascular changes, except for diabetic nephropathy [21], with longer follow up times than our experimental period. Accordingly, it is difficult to determine whether the diabetic state is really associated with a premature atherosclerosis process. On the other hand, in long-term STZ-induced DM rats macroangiopathy in the aorta and coronary blood vessels was not observed, but myocardial damage and ventricular remodelling were found accompanied by LV systolic and diastolic dysfunction $[9,10,14]$. Thus, the effects of Type 1 and 2 DM on the cardiovascular system, especially on cardiac function and myocyte morphology as well as on small coronary arteries seem to be different, although pathological changes in capillary vessels of the heart were commonly observed, and interstitial fibrosis did not appear in either type of DM. However, it is unclear whether or not the experimental results are directly relevant to clinical Type $2 \mathrm{DM}$, so they should be interpreted with care.

\section{References}

1. Hamby RI, Zoneraich S, Sherman L (1974) Diabetic Cardiomyopathy. JAMA 299:1749-1754

2. D'elia JA, Weinrauch LA, Healy RW, Libertino JA, Bradley RF, Leland OS Jr (1979) Myocardial dysfunction without coronary artery disease in diabetic renal failure. Am J Cardiol 43:193-199

3. Shapiro LM (1982) Echocardiographic features of impaired ventricular function in diabetes mellitus. Br Heart J 47: 439-444

4. Kannel WB, McGee DL (1979) Diabetes and cardiovascular disease. The Framingham Study. JAMA 241:2035-2038

5. Manson JE, Colditz GA, Stamnfer MJ et al. (1991) A prospective study of maturity-onset diabetes mellitus and risk of coronary heart disease and stroke in woman. Arch Intern Med 151:1141-1147

6. Factor SM, Minase T, Sonnenblick AH (1980) Clinical and morphological features of human hypertensive-diabetic cardiomyopathy. Am Heart J 99:446-458

7. Factor SM, Okun EM, Minase T (1980) Capillary microaneurysms in the human diabetic heart. $\mathrm{N}$ Engl J Med 302:384-388 
8. Kawaguchi M, Ishihata T, Asakura T et al. (1997) A comparrison of ultrastructural changes on endomyocardial biopsy specimens obtained from patients with diabetes mellitus with and without hypertention. Heart Vessels 12:267-274

9. Litwin SE, Raya TE, Anderson PG, Daugherty S, Goldman S (1990) Abnormal cardiac function in the Streptozotocindiabetic rat changes in active and passive properties of the left ventricle. J Clin Invest 86:481-488

10. Penpargkul S, Schalible T, Yipintsoi T, Scheuer J (1990) The effect of diabetes on performance and metabolism of rat hearts. Circ Res 47:911-921

11. Fein FS, Strobeck JE, Malhotra A, Scheuer J, Sonnenblick EH (1981) Reversibility of diabetic cardiomyopathy with insulin in rats. Circ Res 49:1251-1261

12. Vadulamudi RV, Verma S, Rodgers RL, McNeill JH (1982) The effect of chronic alloxan- and streptozotosin-induced diabetes on isolated rat heart performance. Can J Physiol Pharmacol 60:902-911

13. Verma, Subodh, McNeill JH (1994) Metformin improves cardiac function in isolated streptozotocin-diabetic rat hearts. Am J Physiol 266 :H714-H719

14. Takeda N, Dixon IMC, Hata T, Elimban V, Shah KR, Dhalla NS (1996) Sequence of alterations in subcellular organelles during the development of heart dysfunction in diabetes. Diabetes Res Clin Pract 30[Suppl]:S113-S122

15. Kawaguchi M, Asakura T, Saito F et al.(1999) Changes in diameter size and F-actin expression in the myocytes of patients with diabetes and streptozotocin-induced diabetes model rats. J Cardiol 34:333-339

16. Takeshita M, Ina K, Kitamura H, Shimada T, Nakamura M (1997) Ultrastructural study of capillary and myocytic changes in the masseter and heart of KK-Ay mice. J Electron Microsc 46:413-423

17. Kawano K, Hirashima T, Mori S, Kurosumi M, Saitoh Y, Natori T (1992) Spontaneous long-term hyperglycemic rat with diabetic complications. Otsuka Long-Evans Tokushima fatty (OLETF) strain. Diabetes 41:1422-1428

18. Kawano K, Hirashima T, Mori S, Natori T (1994) OLETF (Otsuka Long-Evance Tokushima Fatty) rat; a new NIDDM rat strain. Diabetes Res Clin Pract 24[Suppl]:S317-S320

19. Ishida K, Mizuno A, Min Z, Sano T, Shima K (1995) Which is the primary etiologic event in Otsuka Long-Evans Tokushima Fatty rats, a model of spontaneous non-insulindependent diabetes mellitus, insulin resistance, or impaired insulin secretion. Metabolism 44:940-945

20. Kosegawa I, Katayama S, Kikuchi C et al. (1996) Metformin decreases blood pressure and obesity in OLETF rats via improvement of insulin resistance. Hypertens Res 19:37-41

21. Mori S, Kawano K, Hirashima T, Natori T (1996) Relationships between diet control and development of spontaneous type II diabetes and diabetic nephropathy in OLETF rats. Diabetes Res Clin Pract 33:145-152
22. Nakamura T, Matsumuro A, Kuribayashi T et al. (1992) Echocardiographic determination of stroke volume during rapid atrial pacing and volume loading in normal rats. Cardiovasc Res 26:765-769

23. Numaguchi K, Egashira K, Takemoto M et al. (1995) Chronic inhibition of nitric oxide synthesis causes coronary microvascular remodeling in rats. Hypertension 26:957962

24. Hsu SM, Raine L, Fanger H (1981) Use of avidin-biotinperoxidase complex $(\mathrm{ABC})$ in immunoperoxidase techniques: a comparison between $\mathrm{ABC}$ and unlabeled antibody (PAP) procedures. J Histochem Cytochem 29:577-580

25. Rodrigues B, Goyal RK, McNeill JH (1986) Effects of hydralazine on streptozotocin-induced diabetic rats: prevention of hyperlipidemia and improvement in cardiac function. J Pharmacol Exp Ther 237:292-299

26. Hsu KL, Tsai CH, Chi Ft et al. (1997) Myocardial mechanics and titin in experimental insulin-resistant rats. Jpn Heart J 38:717-728

27. Dhalla NS, Liu X, Panagia V, Takeda N (1998) Subcellular remodeling and heart dysfunction in chronic diabetes. Cardiovascular Res 40:239-247

28. Poirier P, Garneau C, Bogaty P et al. (2000) Impact of left ventricular diastolic dysfunction on maximal treadmill performance in normotensive subjects with well-controlled type 2 diabetes mellitus. Am J Cardiol 15:473-477

29. Zabalgoitia M, Isumaeil MF, Anderson L, Maklady FA (2001) Prevalence of diastolic dysfunction in normotensive, asymptomatic patients with well-controlled type 2 diabetes mellitus. Am J Cardiol 87:320-323

30. Mizushige K, Li Yao, Noma T et al. (2000) Alteration in left ventricular diastolic filling and accumulation of myocardial collagen at insulin-resistant prediabetic stage of a type II diabetic rat model. Circulation 101:899-907

31. Fein FS (1996) Clinical manifestation of diabetic cardiomyopathy. In: Chatham JC, Forder JR, McNeill JH (eds) The heart in diabetes. Kluwer, Norwell, MA, pp 9-15

32. Akiyama N, Okumura K, Watanabe Y, Hashimoto H, Ito T, Ogawa K et al. (1989) Altered acetylcholine and norepinephrine concentrations in diabetic rat heart. Diabetes 38:231-236

33. Yagi K, Kim S, Wanibuchi H, Yamashita T, Yamamura Y, Iwao H (1997) Characteristics of diabetes, blood pressure, and cardiac and renal complications in Otsuka LongEvance Tokushima Fatty rats. Hypertension 29:728-735

34. Welt K, Weiss J, Koch S, Fitzl G (1999) Protective effects of Ginkgo biloba exact EGb 761 on the myocardium of experimentally diabetic rats II. Ultrastructural and immunohistochemical investigation on microvessels and interstitium. Exp Toxicol Pathol 51:213-222

35. Standl E (1995) Cellular and molecular mechanisms in the macroangiopathy of diabetics. In:Schwartz CJ, Born GVR (eds) Diabetes mellitus and cardiovascular disease. Current Science, San Antonio, pp 124-131 